\section{LOX-1 deficient mice show resistance to zymosan-induced arthritis}

\author{
Kazuhiko Hashimoto, Yutaka Oda, \\ Koichi Nakagawa, Terumasa Ikeda, \\ Kazuhiro Ohtani, Masao Akagi \\ Department of Orthopaedic Surgery, \\ Kindai University Hospital, \\ Osaka-Sayama City, Osaka, Japan
}

\begin{abstract}
Recent data suggest that the lectin-like oxidized low-density lipoprotein (ox-LDL) receptor-1 (LOX-1)/ox-LDL system may be involved in the pathogenesis of arthritis. We aimed to demonstrate the roles of the LOX1/ox-LDL system in arthritis development by using LOX-1 knockout (KO) mice. Arthritis was induced in the right knees of C57Bl/6 wild-type (WT) and LOX-1 KO mice via zymosan injection. Saline was injected in the left knees. Arthritis development was evaluated using inflammatory cell infiltration, synovial hyperplasia, and cartilage degeneration scores at 1,3, and 7 days after administration. LOX-1, ox-LDL, and matrix metalloproteinase-3 (MMP-3) expression in the synovial cells and chondrocytes was evaluated by immunohistochemistry. The LOX-1, ox-LDL, and MMP-3 expression levels in synovial cells were scored on a grading scale. The positive cell rate of LOX-1, ox-LDL, and MMP-3 in chondrocytes was measured. The correlation between the positive cell rate of LOX-1 or ox-LDL and the cartilage degeneration score was also examined. Inflammatory cell infiltration, synovial hyperplasia, and cartilage degeneration were significantly reduced in the LOX-1 KO mice with zymosan-induced arthritis (ZIA) compared to WT mice with ZIA. In the saline-injected knees, no apparent arthritic changes were observed. LOX-1 and ox-LDL expression in synovial cells and chondrocytes were detected in the knees of WT mice with ZIA. No LOX-1 and ox-LDL expression was detected in the knees of LOX-1 KO mice with ZIA or the salineinjected knees of both mice. MMP-3 expression in the synovial cells and chondrocytes was also detected in knees of both mice with ZIA, and was significantly less in the LOX-1 KO mice than in WT mice. The positive cell rate of LOX-1 or ox-LDL and the cartilage degeneration score showed a positive correlation. Our data show the involvement of the LOX-1/ox-LDL system in murine ZIA development. LOX-1-positive synovial cells and chondrocytes are potential therapeutic targets for arthritis prevention.
\end{abstract}

\section{Introduction}

Oxidized low-density lipoprotein (oxLDL) is produced by the oxidation of LDL and plays an essential role in the pathogenesis of atherosclerosis. ${ }^{1}$ Lectin-like ox-LDL receptor-1 (LOX-1) is an important receptor for ox-LDL that has been cloned from cultured bovine aortic endothelial cells, ${ }^{2}$ and has been reported to be expressed in many different cell types, including endothelial cells, ${ }^{3,4}$ vascular smooth muscle cells, ${ }^{5}$ macrophages, ${ }^{6}$ and chondrocytes. ${ }^{7,8}$

Several studies have shown that the LOX-1/ox-LDL system plays a role in the development of cartilage degeneration. Nakagawa et al. first demonstrated LOX-1 expression and the presence of ox-LDL in articular chondrocytes in zymosan-induced arthritis (ZIA). ${ }^{7}$ Moreover, they indicated that rat articular chondrocytes under basal culture environment expressed LOX-1, and that ox-LDL which binds to LOX-1 downregulated the viability of cultured chondrocytes. ${ }^{9}$ The presence of ox-LDL in human synovial fluid ${ }^{10}$ and the co-expression of LOX-1 and ox-LDL in human osteoarthritis cartilage have also been reported. ${ }^{8,11} \mathrm{We}$ previously demonstrated that ox-LDL binding to LOX-1 increased the production of intracellular reactive oxygen species (ROS) in bovine articular chondrocytes (BACs), ${ }^{12}$ induced senescence of articular chondrocytes prematurely with prevention of telomerase activity, ${ }^{13}$ and stimulated a hypertrophic chondrocyte-like phenotype via oxidative stress in cultured BACs. ${ }^{14}$ We also demonstrated previously that the LOX1/ox-LDL system contributed to the pathogenesis of instability-induced and age-related knee osteoarthritis using LOX-1 KO mice. ${ }^{15,16}$ A recent report showed that LOX-1 expression was detected in the chondrocytes of patients with rheumatoid arthritis (RA) and that matrix metalloproteinase-3 (MMP-3) expression was increased by stimulation of ox-LDL. ${ }^{10}$ Ishikawa et al. also reported that the LOX-1 signal has a possibility as a biomarker and therapeutic target in human RA. ${ }^{17}$ Although these findings suggest that the LOX-1/ox-LDL system plays multiple roles in promoting arthritis, whether LOX-1 deficiency affects the development of arthritis remains unknown. Therefore, we investigated to clarify the roles of the LOX-1/ox-LDL system in the development of arthritis by using LOX-1 KO mice. Zymosan activates the alternative complement pathway, at which point macrophages that phagocytose zymosan are activated. ${ }^{18}$ The activated macrophage produces inflammatory mediators such as interleukin-1 (IL-1) and releases lysosomes and ROS. ${ }^{19}$ As a result, hypervascular permeability and leukocyte infil-
Correspondence: Kazuhiko Hashimoto, Department of Orthopaedic Surgery, Kindai University Hospital, 377-2 Ohno-Higashi, Osaka-Sayama City, Osaka 589-8511, Japan. Tel. +81.072.366-0221 - Fax: +81.072.366-0206. E-mail: hazzhiko@med.kindai.ac.jp

Key words: Lectin-like oxidized low-density lipoprotein receptor-1 (LOX-1); oxidized lowdensity lipoprotein (ox-LDL); mouse; knee; zymosan-induced arthritis.

Contributions: $\mathrm{KH}, \mathrm{MA}$, study conception and design; $\mathrm{KH}, \mathrm{YO}$, data acquisition; $\mathrm{KH}, \mathrm{MA}$, $\mathrm{KN}$, TI, OK, analysis and interpretation of data. All the authors drafted the manuscript and approved the final version.

Conflict of interest: The authors declare no conflict of interest.

Acknowledgments: We thank Mr. Katsumi Okumoto (Department of Life Science, Kindai University Hospital) for providing technical assistance, which helped in performing the experiments. We also would like to thank Editage (www.editage.jp) for English language editing.

Received for publication: 2 September 2017. Accepted for publication: 8 January 2017.

This work is licensed under a Creative Commons Attribution-NonCommercial 4.0 International License (CC BY-NC 4.0).

CCopyright K. Hashimoto et al., 2018 Licensee PAGEPress, Italy

European Journal of Histochemistry 2018; 62:2847 doi:10.4081/ejh.2018.2847

tration can occur. When zymosan is injected into the joint cavity, arthritic changes are induced by the mechanism described above. ZIA is often used as an animal model of arthritis and has some similarities to RA. ${ }^{20,21}$ In light of these facts, we selected this particular animal model to clarify the roles of the LOX-1/ox-LDL system during arthritis development in LOX-1 KO mice with ZIA.

\section{Materials and Methods}

\section{Mice}

LOX-1+/+, C57BL/6 Jcl mice (WT) were provided by Nihon CLEA (Tokyo, Japan). LOX-1-/-, C57BL/6 Jcl mice (KO) were originally generated by Sawamura et al., ${ }^{22}$ and were provided by the National Cerebral and Cardiovascular Center (Osaka, Japan). Pups were genotyped by Southern blotting using an external probe. ${ }^{22}$ Mice were housed in cages with free access 

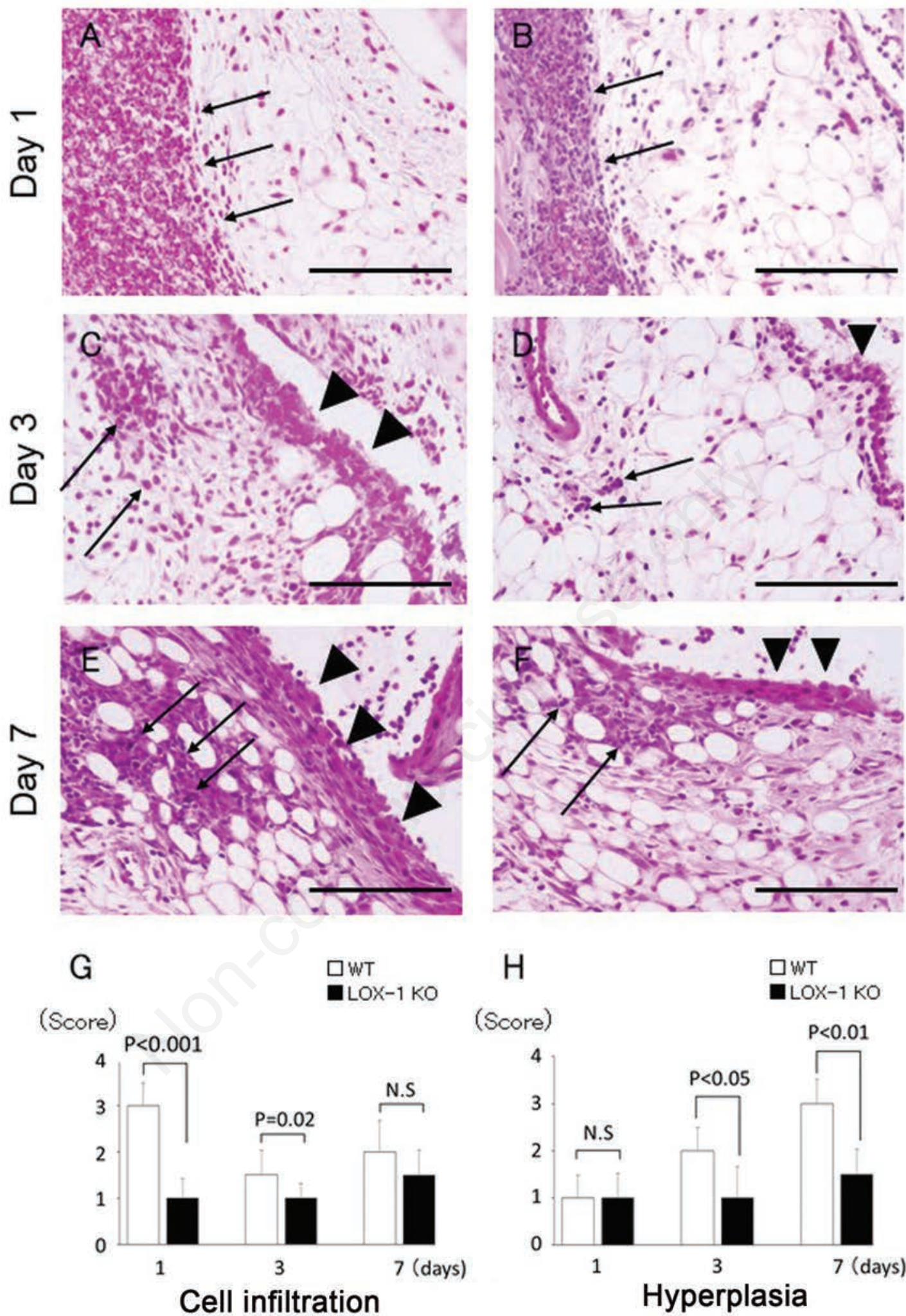

$\mathrm{H}$

\section{口WT}

(Score)

LOX $-1 \mathrm{KO}$

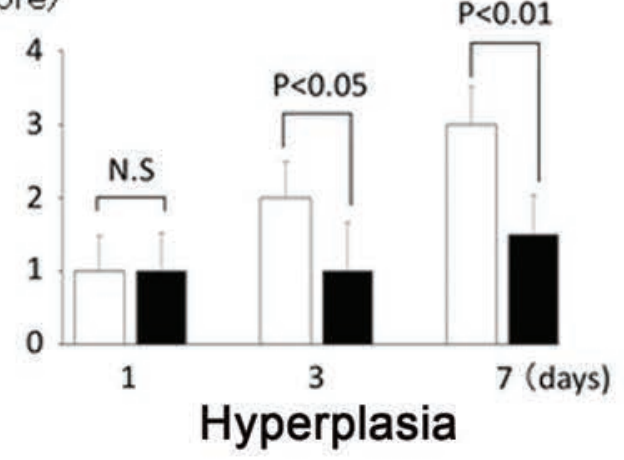

Figure 1. The panels show representative synovium in zymosan-injected group stained with $\mathrm{H} \& \mathrm{E}$ (A-F) at $400 \times$ magnification. Synovium of WT mice (A, C, E) and LOX-1 KO mice (B, D, F) at day 1, 3, and 7 after the zymosan injection. The graphs show the score of inflammatory cell infiltration $(G)$ and synovial hyperplasia $(H)$ in zymosan-injected groups at each experimental time. Inflammatory cell infiltration is observed less in the synovium of LOX-1 KO mice (B, D) than in that of WT mice (A, C) at day 1 and 3. Synovial hyperplasia was also observed less in the synovium of LOX-1 KO mice (D, F) than in that of WT mice (C, E) at day 3 and 7. Arrows show the infiltrated inflammatory cells to synovium. Arrowheads show the synovial hyperplasia. Data are presented as mean + SD ( $n=10$, in each group); significant difference $(P<0.05$, Student's $t$-test $)$ are indicated between the score of WT and that of LOX$1 \mathrm{KO}$ mice). N.S, not significant. Scale bars: $100 \mu \mathrm{m}$. 

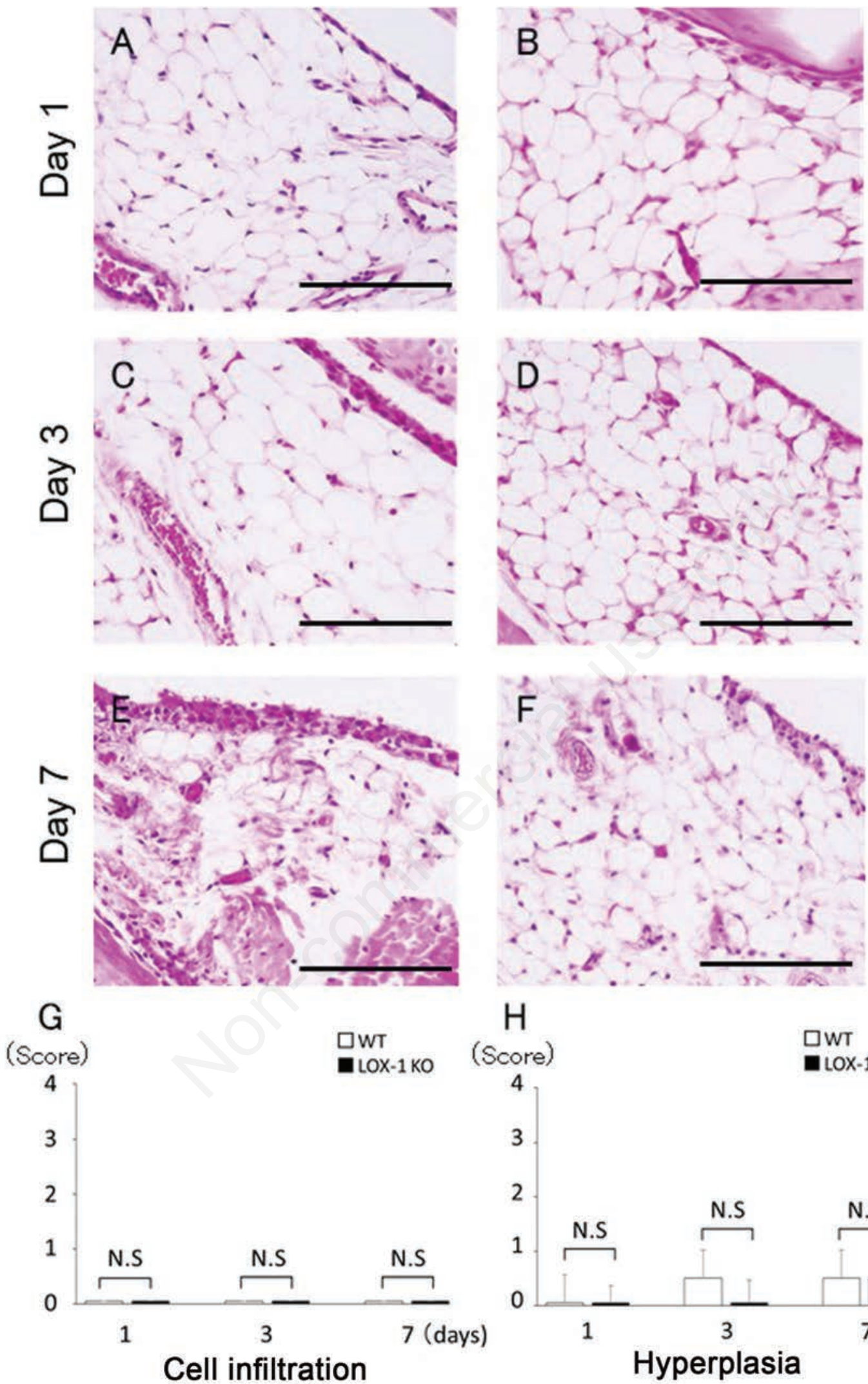

$\mathrm{H}$
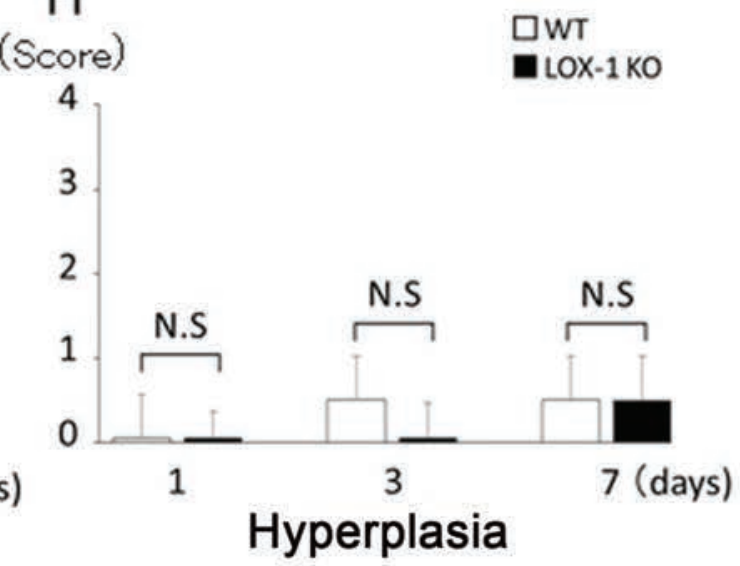

Figure 2. The panels show representative synovium in saline-injected group stained with $\mathrm{H} \& \mathrm{E}$ (A-F) at $400 \times$ magnification. Synovium of WT mice (A, C, E) and LOX-1 KO mice (B, D, F) at day 1, 3, and 7 after the saline injection. No inflammatory cell infiltration was observed in the saline-injected knees of both WT (A, C, E) and LOX-1 KO mice (B, D, and F). No obvious synovial hyperplasia was observed in the saline-injected knees of both WT (A, C, E) and LOX-1 KO mice (B, D, F). The graphs show the score of inflammatory cell infiltration $(G)$ and synovial hyperplasia $(H)$ in saline-injected groups at each experimental time. Data are presented as mean + SD $(n=10$, in each group); $\mathrm{P}<0.05$ was regarded as a significant difference (Student's t-test). N.S, not significant. Scale bars: $100 \mu \mathrm{m}$. 
to food and water in a temperature-controlled room with a 12-h dark/12-h light cycle. All animal experiments were performed according to protocols approved by the Animal Care and Use Committee of the Kindai University Hospital.

\section{Zymosan-induced arthritis model}

ZIA model mice were prepared in accordance with a method previously described. ${ }^{7}$ WT and LOX-1 KO mice (all 9-week-old males) were used for arthritis evaluation. The skin was incised longitudinally at the lateral margin of the patella, and the capsule and patellar tendon were exposed. The mice in the experimental group were subjected to intraarticular injections of zymosan (180 $\mu \mathrm{g} / 6 \mu \mathrm{L}$; Sigma-Aldrich, St. Louis, MO, USA) into the right knee joint from the lateral side using a 30 -g needle attached to a $10-\mu \mathrm{L}$ syringe (HAMILTON, SigmaAldrich). In the same way, sterile saline was also injected into the left knee as a negative control. The skin was closed with 7-0 nylon. Mice were killed by cervical dislocation at 1,3 , and 7 days after administration. Knee joints obtained from these mice were used for histologic analyses. All mice were kept in accordance with the guidelines of the Animal Research Committee, Graduate School of Medicine, Kindai University.
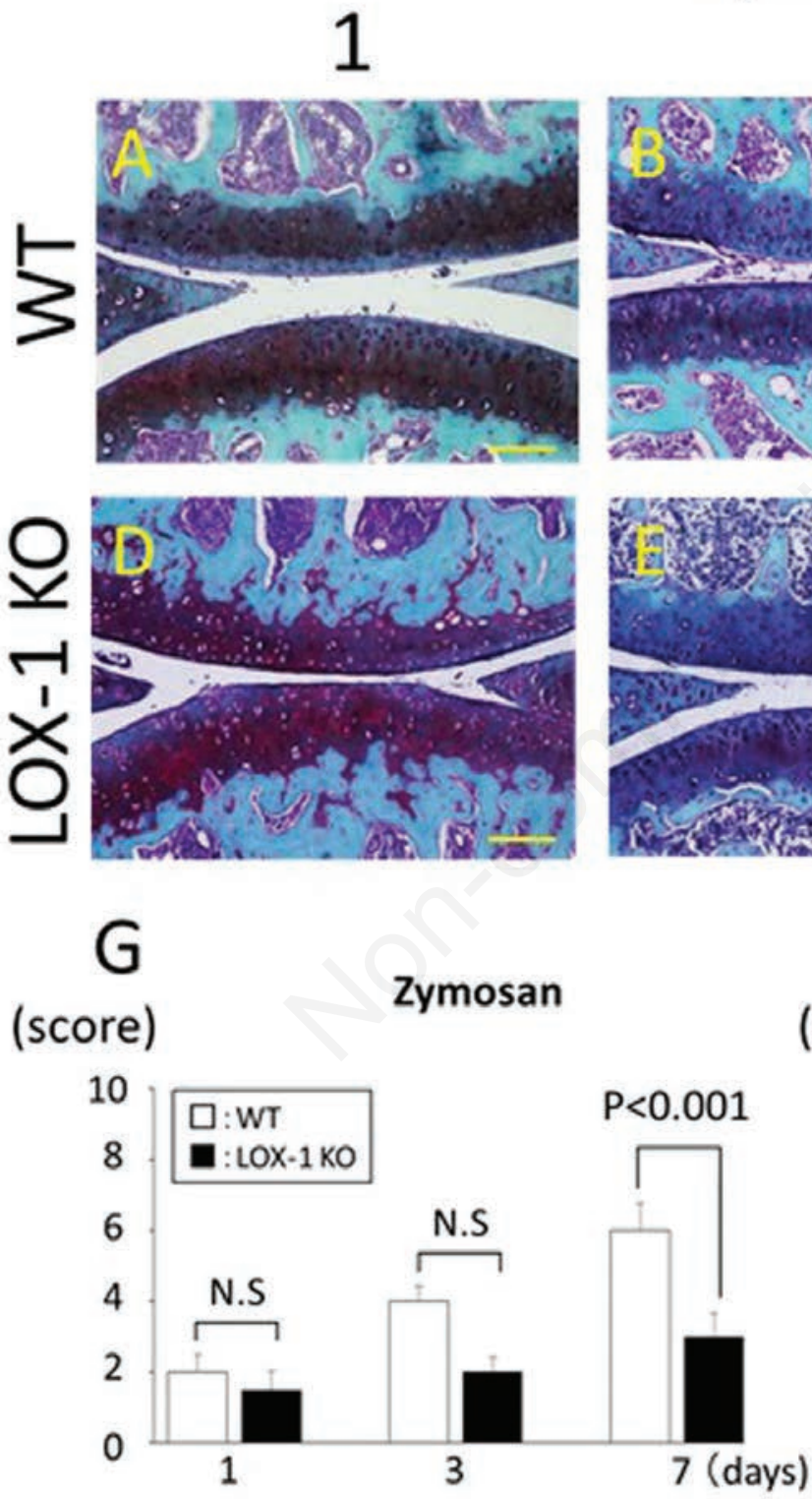

\section{Zymosan}

3
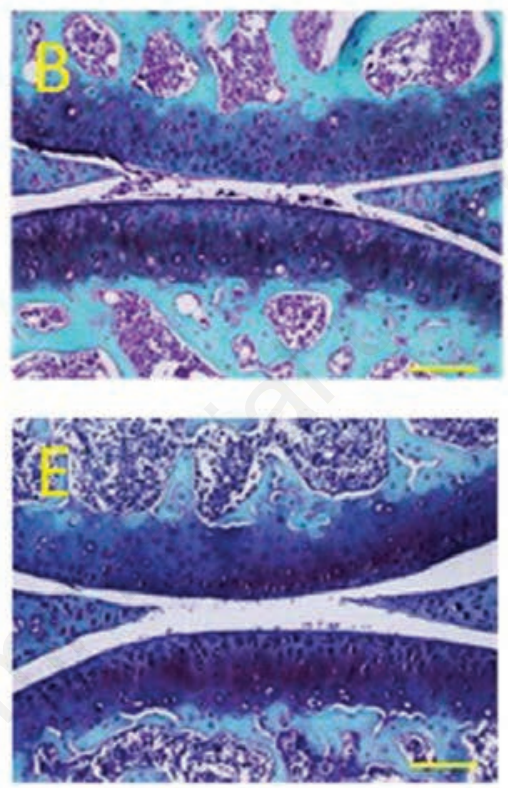

\section{7 (days)}
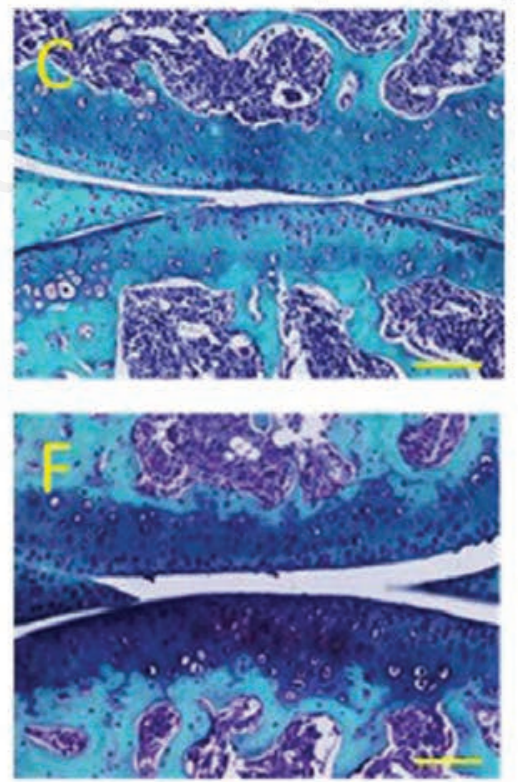

\section{Saline}

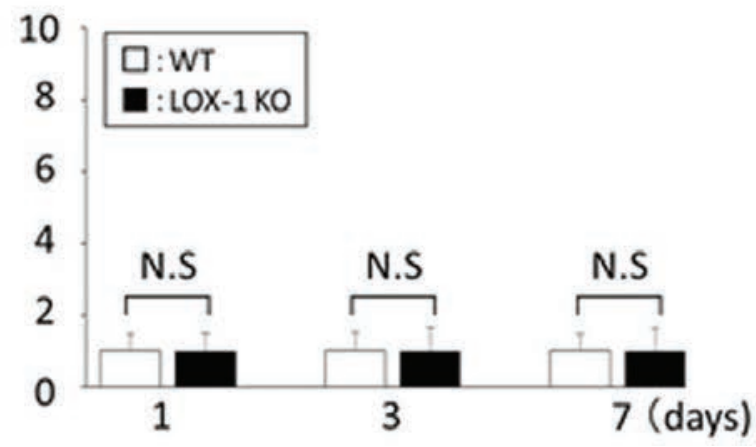

Figure 3. The panels show representative articular cartilage staining with SFO (A-F). Articular cartilage of WT mice at day 1, 3, and 7 after zymosan injection (A-C). Articular cartilage of LOX-1 KO mice at day 1, 3, and 7 after zymosan injection (D-F). All specimens are in the sagittal direction. The graphs show the score of cartilage degeneration in the zymosan-injected knees $(G)$, and saline-injected knees $(H)$ at each experimental time. Cartilage degeneration was lesser in the cartilage of LOX-1 KO mice (D-F) than in WT mice (A-C) during all experimental times. Data are presented as mean $+S D(n=10$, in each group); $P<0.05$ was regarded as a significant difference (Student's $t-$ test). N.S, not significant. Scale bars: $100 \mu \mathrm{m}$. 


\section{Zymosan}
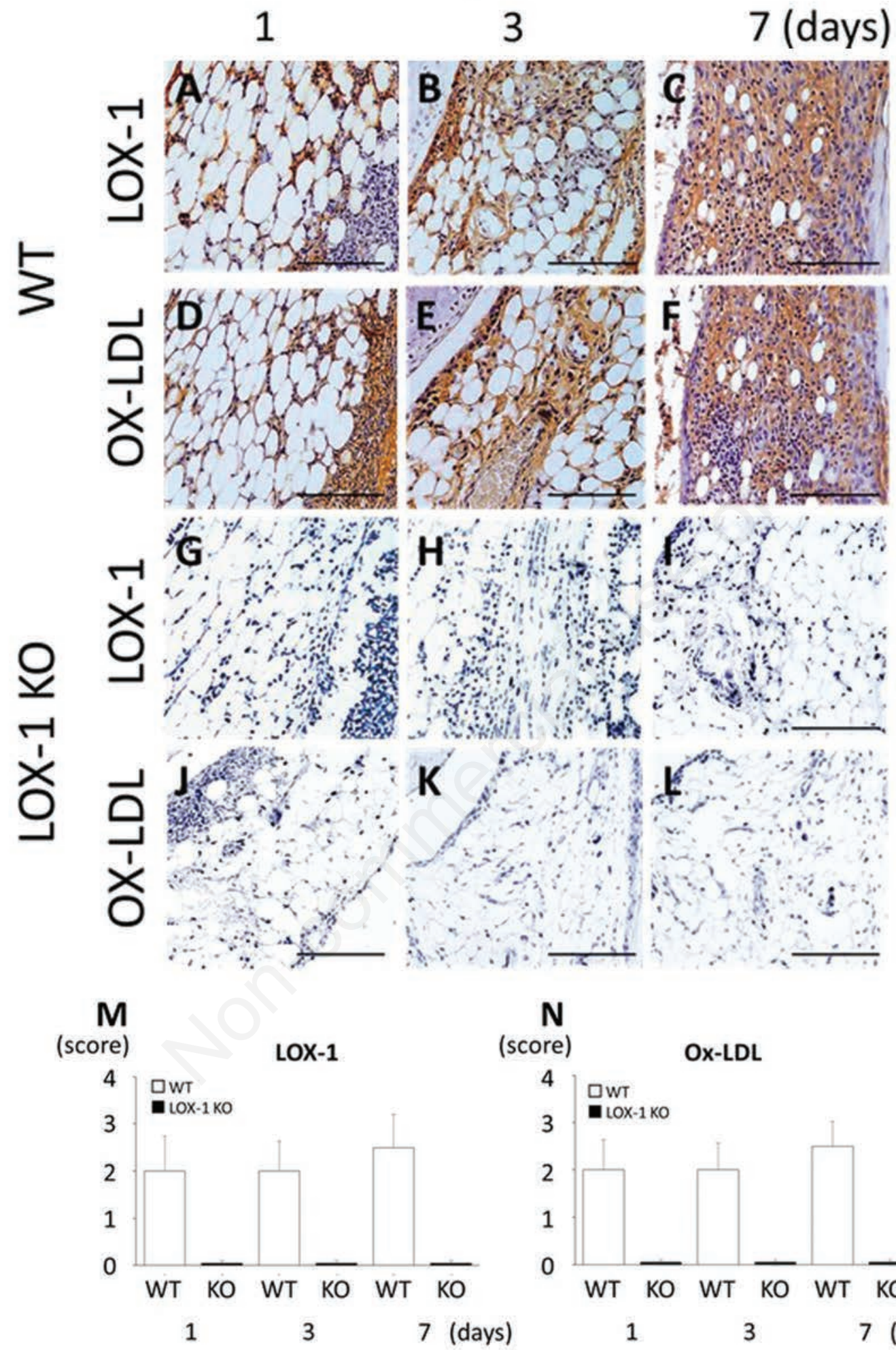

$\mathbf{N}$

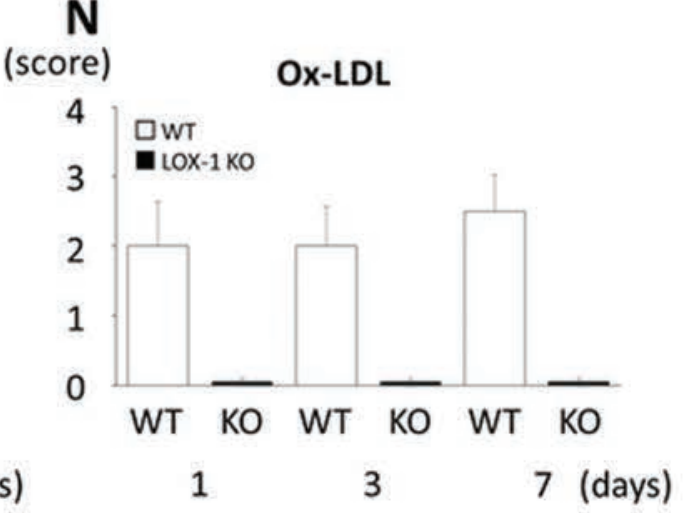

Figure 4. The panels show representative synovial tissues with immunostaining for LOX-1 (A-C) and ox-LDL (D-F) at 200× magnification. LOX-1 expression in the synovial cells of WT mice at day 1, 3 and 7 after zymosan injection (A-C). Ox-LDL expression in the synovial cells of WT mice at day 1, 3 and 7 after zymosan injection (D-F). LOX-1 expression in the synovial cells of LOX-1 KO mice at day 1,3 and 7 after zymosan injection (G-I). Ox-LDL expression in the synovial cells of LOX-1 KO mice at 1, 3 and 7 days after injection of zymosan (J-L). The graphs show the positive cell score of LOX-1 (M) and ox-LDL (N) expression in the synovium of WT and LOX-1 KO mice at each experimental time. The antibodies used in the study were rabbit anti-mouse LOX-1 polyclonal antibody and rabbit antimouse ox-LDL polyclonal antibody. Data are presented as mean + SD ( $n=10$, in each group). Scale bars: $100 \mu \mathrm{m}$. 


\section{Histologic analysis}

Specimens of whole mice knee joints were dissected and fixed overnight in $4 \%$ paraformaldehyde in phosphate buffered saline (PBS) (pH 7.4). After decalcification with $10 \%$ ethylenediaminetetraacetic acid $(\mathrm{pH} 7.4)$ at $4^{\circ} \mathrm{C}$ for 14 days, the samples were embedded in paraffin and cut into 4- $\mu \mathrm{m}$ sections along the sagittal plane. Sections were stained with hematoxylin and eosin (H\&E) and Safranin-O (SFO) in order to evaluate synovial inflammation and cartilage degeneration. The sections deparaffinized in xylene and dehydrated using an ethanol gradient were immersed in SFO solution (Sigma-Aldrich) for $10 \mathrm{~min}$. Sections were washed in PBS for 5 min and then immersed in 1\% Fast Green solution (Wako, Osaka, Japan) for 5 min. ${ }^{23}$ The joint inflammatory pathology was scored on a scale of 0-3 based on the degree of cellular infiltration into the synovium and synovial hyperplasia. ${ }^{24}$ Inflammatory cells in combined synovial images $(200 \times$ magnification) were counted with a cell counting program described in a previous study. ${ }^{24}$
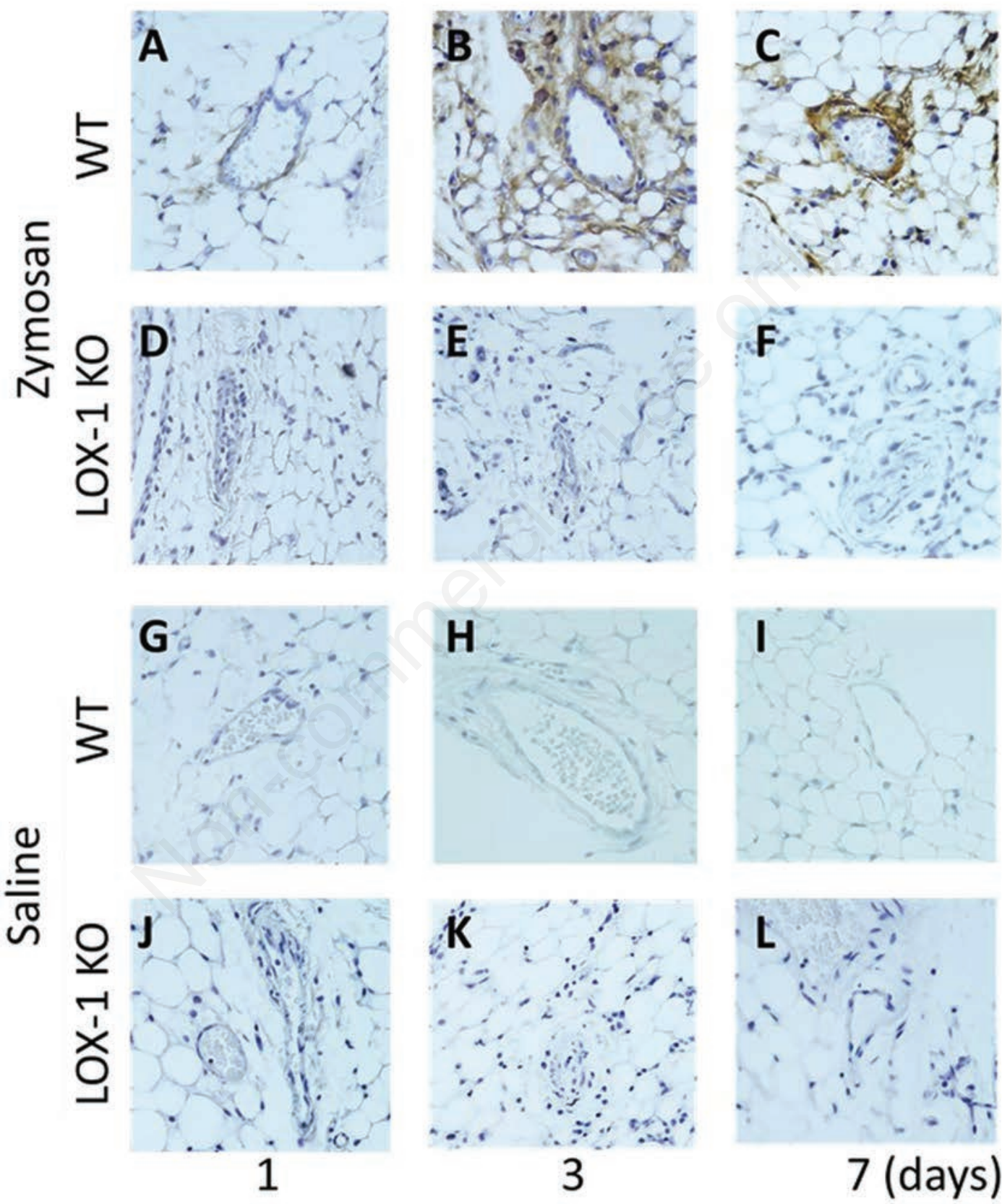

\footnotetext{
Figure 5. Representative synovial vascular endothelium with LOX-1 immunostaining (A-L). LOX-1 expression in the synovial vascular endothelium of WT mice at day 1,3 and 7 after zymosan injection (A-C) at 400x magnification. LOX-1 expression in the synovial vascular endothelium of LOX-1 KO mice at day 1, 3, and 7 after zymosan injection (D-F). LOX-1 expression in the synovial vascular endothelium of WT mice at day 1, 3, and 7 after saline injection (G-I). LOX-1 expression in the synovial vascular endothelium of LOX-1 KO mice at day 1, 3, and 7 after saline injection (J-L). The expression is observed only in the synovial vascular endothelium of WT mice with zymosaninjected groups during all the experimental times (A-C). The antibodies used in the study were rabbit anti-mouse LOX-1 polyclonal antibody and rabbit anti-mouse ox-LDL polyclonal antibody.
} 
Cartilage degeneration was also scored on a scale of $0-11 .{ }^{25}$ Equal numbers of slides ( $\mathrm{n}=$ 10 , in each, at $200 \times$ magnification) from all groups were analyzed by two independent observers (KH and YO). These analyses were performed on slides decoded by another researcher.

\section{Immunohistochemistry}

To visualize the LOX-1, ox-LDL, and MMP-3 in knee joint tissues during arthritis progression, immunostaining was performed. Tissue sections near those used for evaluating synovial inflammation and cartilage degeneration were deparaffinized in xylene and rehydrated with an ethanol gradient. Endogenous peroxidase activity was blocked by immersion in $3 \% \mathrm{H}_{2} \mathrm{O}_{2}$ in methanol for $5 \mathrm{~min}$. Then, the sections were incubated in blocking solution $(3 \%$ bovine serum in PBS) for $15 \mathrm{~min}$ at room temperature, followed by incubation with a diluted primary antibody overnight at $4{ }^{\circ} \mathrm{C}$. The primary antibodies used were: rabbit antimouse LOX-1 polyclonal antibody (Abcam; 1:100 dilution in 0.1\% Triton-Trisbuffered saline (TBS) containing 10\% Block Ace; DS Pharma Biomedical, Osaka, Japan), rabbit anti-mouse ox-LDL polyclonal antibody (LSL, Tokyo; 1:100 dilution in $0.1 \%$ Triton-TBS containing $10 \%$ Block Ace; DS Pharma Biomedical), and rabbit anti-mouse MMP-3 polyclonal antibody (Abcam; 1:500 dilution in 0.1\% Triton-TBS containing 10\% Block Ace; DS Pharma Biomedical). After primary antibody incubation, the LOX-1, ox-LDL, and MMP-3-labeled sections were washed in PBS and incubated with a horseradish peroxidase-conjugated donkey anti-rabbit $\mathrm{IgG}$ secondary antibody (Santa Cruz Biotechnology, Santa Cruz, CA, USA; 1:1,000 dilution) at room temperature for 30 min. Then, the sections were washed with PBS, immersed in a diaminobenzidine solution (Agilent Technologies, Santa Clara, CA, USA) at room temperature for $10 \mathrm{~min}$ to visualize immunoreactivity, and counterstained with hematoxylin. The expression levels of LOX-1, ox-LDL, and MMP-3 in synovial cells were scored on a scale 0 to 3 (where $0=$ no staining and $3=$ greatest staining) as previously described. ${ }^{26}$ The positive cell rate of LOX-1, ox-LDL, and MMP-3 in chondrocytes was also measured as previously described. ${ }^{15,16}$ The percentage of positive cells was measured as the number of stained cells relative to the number of total chondrocytes (mean [range]; 88.5 [78-102]) in the medial tibial plateau. The correlation between the cartilage degeneration score and the positive LOX-1 or ox-LDL cell rate in chondrocytes was also examined.

\section{Statistical analysis}

All data have been presented as the mean \pm standard deviation. The scores of each group were compared using Student's $t$-test. P-values less than 0.05 were considered significant. All data were analyzed with Stat Mate (Atms, Tokyo, Japan) software for Windows, version 4.01.15,16 The correlation between the LOX-1 or ox-LDL positive cell rate and the cartilage degeneration score was examined using Pearson's correlation (Excel 2010, Microsoft, Tokyo, Japan).

\section{Results}

\section{Inflammatory cell infiltration and synovial hyperplasia}

We performed H\&E staining in order to evaluate inflammatory cell infiltration and hyperplasia. At 1 day after zymosan injection, inflammatory cell infiltration was most apparent in both the WT and LOX-1 KO mice (Figure $1 \mathrm{~A}, \mathrm{~B}$ ). The inflammatory cell infiltration in the LOX-1 KO mice (Figure 1 $\mathrm{B}, \mathrm{D}, \mathrm{F})$ was less than that in the WT mice (Figure $1 \mathrm{~A}, \mathrm{C}, \mathrm{E})$. In the saline-injected knees, inflammatory cell infiltration was not observed in both the WT and LOX-1 $\mathrm{KO}$ mice (Figure $2 \mathrm{~A}-\mathrm{F}$ ). The inflammatory cell infiltration scores in the WT and LOX$1 \mathrm{KO}$ mice at 1, 3, and 7 days after zymosan injection were $3.0 \pm 0.48$ and $1.0 \pm 0.48$, $1.5 \pm 0.53$ and $1.0 \pm 0.32$, and $2.0 \pm 0.67$ and $1.5 \pm 0.53$, respectively (Figure $1 \mathrm{G}$ ). The inflammatory cell infiltration score in the LOX-1 KO mice was significantly lower than that in the WT mice at 1 and 3 days after zymosan injection (Figure 1G; $\mathrm{P}<0.001, \mathrm{P}=0.02, \mathrm{n}=10$ in each group, respectively). The inflammatory cell infiltration score at 7 days after zymosan injection showed no significant difference between the WT and LOX-1 KO mice. (Figure $1 \mathrm{G}, \mathrm{P}=0.08, \mathrm{n}=10$ ). All inflammatory cell infiltration scores in saline-injected knees were 0 in both the WT and LOX-1 $\mathrm{KO}$ mice (Figure $2 \mathrm{G}, \mathrm{n}=10$ in each group). Following zymosan injection, synovial hyperplasia was also observed in both the WT and LOX-1 KO mice, but was less in the LOX-1 KO mice than in the WT mice (Figure $1 \mathrm{~A}-\mathrm{F}$ ). In the saline-injected knees, no apparent synovial hyperplasia was observed in either the WT mice or the LOX$1 \mathrm{KO}$ mice (Figure $2 \mathrm{~A}-\mathrm{F}$ ). The synovial hyperplasia scores in the WT and LOX-1 $\mathrm{KO}$ mice at 1,3, and 7 days after zymosan injection were $1.0 \pm 0.48$ and $1.0 \pm 0.52$, $2.0 \pm 0.48$ and $1.0 \pm 0.67$, and $3.0 \pm 0.51$ and $1.5 \pm 0.53$, respectively (Figure $1 \mathrm{H}$ ). Although there was no significant difference in the synovial hyperplasia score between the WT and LOX-1 KO mice at 1 day (Figure $1 \mathrm{H}, \mathrm{P}=1.0, \mathrm{n}=10$ ), the score in the LOX-1 KO mice was significantly lower than that in the WT mice at 3 and 7 days (Figure $1 \mathrm{H} ; \mathrm{P}<0.001, \mathrm{P}<0.001, \mathrm{n}=10$ in each group, respectively). In the salineinjected knees, synovial hyperplasia scores in the WT and LOX-1 KO mice at 1, 3, and 7 days were $0 \pm 0.52$ and $0 \pm 0.32,0.5 \pm 0.53$ and $0 \pm 0.42$, and $0.5 \pm 0.53$ and $0.5 \pm 0.53$, respectively (Figure $2 \mathrm{H}$ ).

\section{Cartilage degeneration}

We performed SFO staining to evaluate cartilage degeneration. Cartilage degeneration was increased according to the exposure time in both the WT and LOX-1 KO mice (Figure $3 \mathrm{~A}-\mathrm{F}$ ). Cartilage degeneration was less in the LOX-1 KO mice than in the WT mice. The cartilage degeneration scores in the WT and LOX-1 KO mice at 1 and 3 days were $2.0 \pm 0.48$ and $4.0 \pm 0.42$, and $1.5 \pm 0.53$ and $2.0 \pm 0.42$, respectively. There was no significant difference between the cartilage degeneration score in the WT and LOX-1 KO mice at 1 and 3 days $(\mathrm{P}=0.74$, $\mathrm{P}=0.12, \mathrm{n}=10$ in each group, respectively). At day 7 , the cartilage degeneration scores in the WT and LOX-1 KO mice were $6.0 \pm 0.79$ and $3.0 \pm 0.67$, and the score in the LOX-1 KO mice was significantly lower than that in the WT mice (Figure 3G; $\mathrm{P}<0.001, \mathrm{n}=10)$. In the saline-injected knees, no apparent cartilage degeneration was observed in either the WT mice or the LOX-1 KO mice (data not shown). In the saline-injected knees, the cartilage degeneration scores in the WT and LOX-1 KO mice were $1.0 \pm 0.48$ and $1.0 \pm 0.52,1.0 \pm 0.54$ and $1.0 \pm 0.65$, and $1.0 \pm 0.47$ and $1.0 \pm 0.63$ at 1,3 , and 7 days, respectively (Figure $3 \mathrm{H}$ ). There was no significant difference between the scores in the WT and LOX-1 KO mice in the saline-injected group (Figure $3 \mathrm{H} ; \mathrm{P}=1.0$, $\mathrm{P}=1.0, \mathrm{P}=1.0, \mathrm{n}=10$ in each group, respectively).

\section{The LOX-1 and ox-LDL expression in synovial cells}

We evaluated the expression of LOX-1 and ox-LDL in synovial cells immunohistochemically. In the synovial cells of the WT mice, LOX-1 and ox-LDL expression was observed at 1, 3, and 7 days after zymosan injection (Figure $4 \mathrm{~A}-\mathrm{F}$ ). In the LOX-1 KO mice with ZIA, no LOX-1 or ox-LDL expression was observed during the entire experimental period (Figure $4 \mathrm{G}-\mathrm{L}$ ). In the saline-injected knees of WT mice, no expression was observed during the entire experimental period (data not shown). In the saline-injected knees of LOX-1 KO mice, no LOX-1 or ox-LDL expression was observed during the entire experimental period (data not shown). In the WT mice 
1

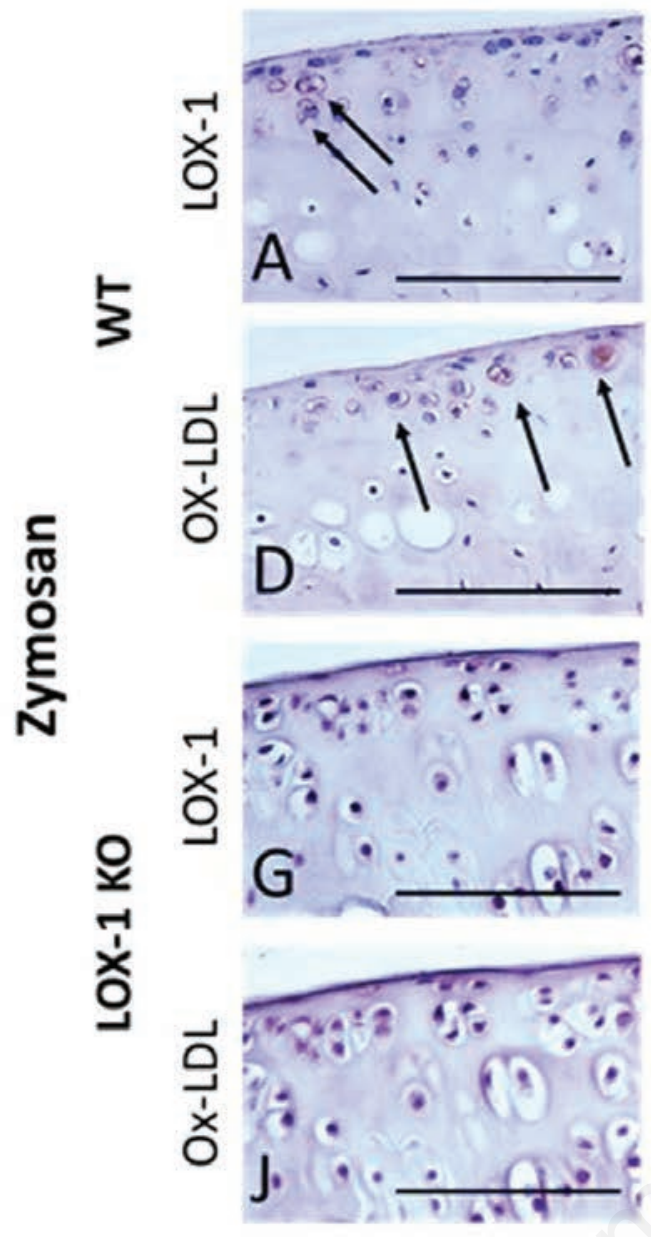

M

(score)

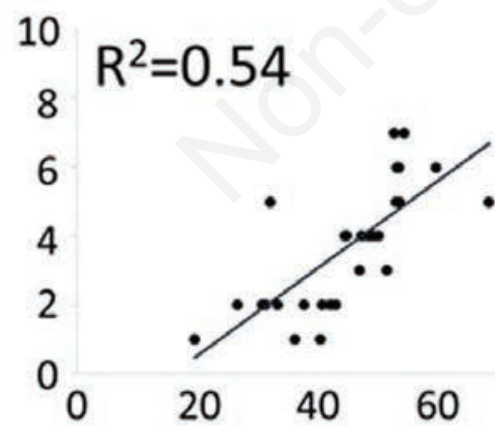

3
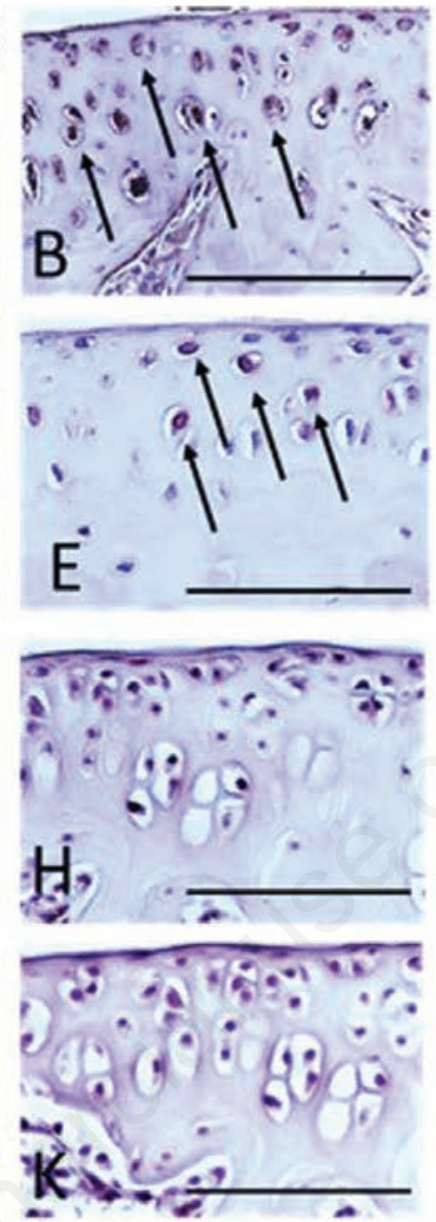

N
7 (days)
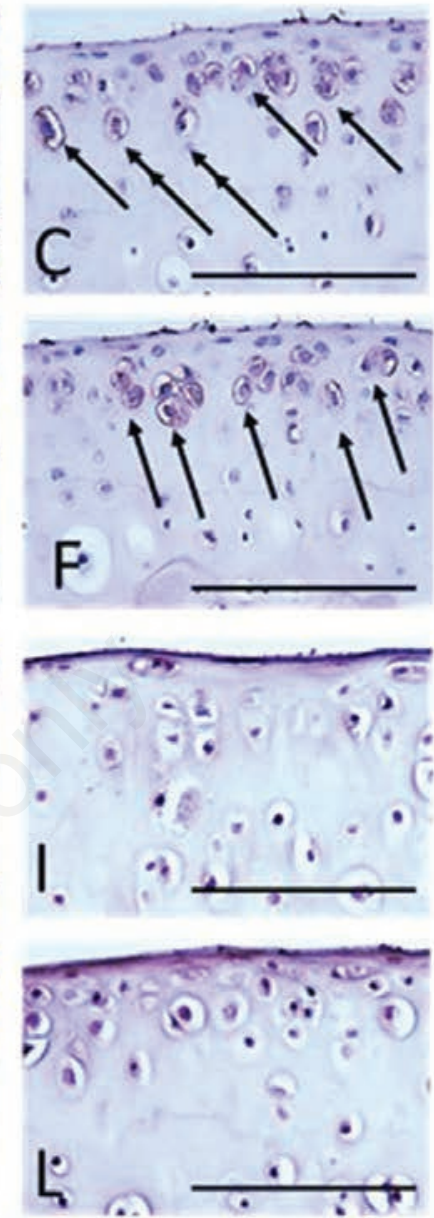

Ox-LDL

(score)

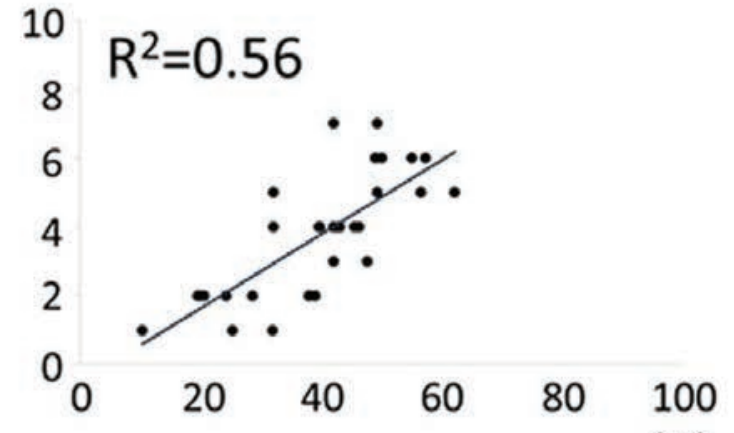

Figure 6. The panels show representative tibial cartilage immunostaining of LOX-1 and ox-LDL (A-L) at 400× magnification. LOX-1 expression in the cartilage of WT mice at day 1, 3 and 7 after zymosan injection (A-C). Ox-LDL expression in the cartilage of WT mice at day 1, 3 and 7 after zymosan injection (D-F). LOX-1 expression in the cartilage of LOX-1 KO mice at day 1, 3 and 7 after zymosan injection (G-I). Ox-LDL expression in the cartilage of LOX-1 KO mice at day 1, 3 and 7 after zymosan injection (J-L). Although LOX-1 and oxLDL positive cells are observed in WT mice (A-F), no positive cells are observed in LOX-1 KO mice (G-L) during all the experimental time. The graphs show the correlation between the positive cell rate for LOX-1 (M), ox-LDL $(\mathrm{N})$ and the cartilage degeneration score in WT mice. The positive correlation is observed between positive cell rate for LOX-1 or ox-LDL in chondrocytes and the cartilage degeneration score. Arrows show the LOX-1 or ox-LDL positive chondrocytes. The antibodies used were rabbit anti-mouse LOX-1 polyclonal antibody and rabbit anti-mouse ox-LDL polyclonal antibody. Scale bars: $100 \mu \mathrm{m}$. 
with ZIA, the positive cell scores of LOX-1 and ox-LDL were $2.0 \pm 0.74$ and $2.0 \pm 0.63$, $2.0 \pm 0.63$ and $2.0 \pm 0.57$, and $2.5 \pm 0.53$ and $2.5 \pm 0.70$ at 1,3 , and 7 days, respectively (Figure $4 \mathrm{M}, \mathrm{N}$ ).

\section{LOX-1 expression in synovial vascu- lar endothelium}

LOX-1 expression was observed in the synovial vascular endothelium of the WT mice with ZIA (Figure 5 A-C). No expression of LOX-1 was found in the synovial vascular endothelium of the LOX-1 KO mice with ZIA (Figure $5 \mathrm{D}-\mathrm{F}$ ). In the salineinjected knees, no expression was observed in any of the mice (Figure 5 G-L).

\section{LOX-1 and ox-LDL expression in articular chondrocytes}

During the entire experimental period in WT mice with ZIA, LOX-1 and ox-LDL expression was observed in chondrocytes (Figure 6 A-F). No LOX-1 and ox-LDL expression was observed in LOX-1 KO mice with ZIA (Figure $6 \mathrm{G}-\mathrm{L}$ ). In the salineinjected knees of the WT and LOX-1 KO mice, no LOX-1 or ox-LDL expression was observed (data not shown). The cartilage degeneration score and positive cell rate of LOX-1 and ox-LDL showed a positive correlation (Figure $6 \mathrm{M}, \mathrm{N} ; \mathrm{R}^{2}=0.54, \mathrm{R}^{2}=0.56$, $\mathrm{n}=30$ in each, respectively,).

\section{MMP-3 expression in synovial cells and articular chondrocytes}

We evaluated MMP-3 expression in synovial cells and articular chondrocytes immunohistochemically. In the synovial cells of the WT mice, MMP-3 expression was observed at 1,3 , and 7 days after zymosan injection (Figure $7 \mathrm{~A}-\mathrm{C}$ ). In the synovial cells of LOX-1 KO mice, MMP-3 expression was also observed after zymosan injection during the entire experimental period (Figure 7 D-F). MMP-3 expression in the synovial cells of the LOX-1 KO mice was less than that in WT mice. In the salineinjected knees of both the WT and LOX-1 $\mathrm{KO}$ mice, no MMP-3 expression was observed during the entire experimental period (data not shown). In the WT mice, MMP-3 positive scores were $1.5 \pm 0.70$, $2.0 \pm 0.63$, and $2.5 \pm 0.82$ at 1,3 , and 7 days after zymosan injection (Figure $7 \mathrm{G}$ ). In the LOX-1 KO mice, MMP-3 positive scores after zymosan injection were $1.0 \pm 0.70$, $1.5 \pm 0.70$, and $1.5 \pm 0.70$ at 1,3 and 7 days, respectively (Figure 7G). There was no significant difference between the WT and LOX-1 KO mice in terms of the MMP-3 positive scores at 1 and 3 days (Figure 7G; $\mathrm{P}=0.127, \mathrm{P}=0.108, \mathrm{n}=10$ in each, respectively). At 7 days, the MMP-3-positive score of the LOX-1 KO mice was significantly lower than that of the WT mice
(Figure 7G; $\mathrm{P}=0.009, \mathrm{n}=10$ ).

MMP-3 expression in chondrocytes was also observed in both mice after zymosan injection (Figure 8 A-F). MMP-3 expression in the chondrocytes of the LOX$1 \mathrm{KO}$ mice was less than that in the WT mice. In the WT mice, the positive cell rate of MMP-3 was 34.2 (20.2-48.2), 26.4 (22.0$30.8)$ and $41.6(33.2-50.0)$ [mean (range)]: $95 \%$ confidence interval, $\mathrm{CI}]$ at 1,3 , and 7 days after zymosan injection. In the LOX-1 $\mathrm{KO}$ mice, the positive cell rate of MMP-3 was 9.8 (4.52-15.1), 11.3 (8.91-13.69) and 16.4 (12.97-19.83) [mean (range): 95\% CI] at 1,3 , and 7 days after zymosan injection (Figure $8 \mathrm{G}$ ), and the positive cell rate of MMP-3 in chondrocytes was significantly lower than that of the WT mice (Figure 8G; $\mathrm{P}=0.005, \mathrm{P}<0.001, \mathrm{P}<0.001, \mathrm{n}=10$ in each group, respectively). In the saline-injected knees, no MMP-3 expression was observed in any of the mice (data not shown).

\section{Discussion}

In the current study, we investigated the roles of the LOX-1/ox-LDL system in the development of arthritis using LOX-1 KO mice with ZIA. Decreased synovitis and cartilage degeneration were observed as a result of LOX-1 knockout, and the roles of the LOX-1/ox-LDL system during the development of arthritis in vivo were clarified. Previous studies reported that when LOX-1 and its ligand bind in vascular endothelial cells, the vascular endothelium expresses cell adhesion molecules such as ICAM-1, VCAM-1, and MCP-1 which is a macrophage chemotactic factor that promotes the adhesion and infiltration of leukocytes to vascular endothelial cells. ${ }^{27}$ These LOX-1 functions are believed to play an important role in the early stage of arteriosclerotic lesion formation. Zymosan is known to enhance the production of IL-1 $\beta$ from macrophages and neutrophils, and IL$1 \beta$ is also known to enhance LOX-1 expression. $^{28}$ A previous report suggested that LOX-1 expressed in the synovial vascular endothelium plays an important role in the leukocyte infiltration process. ${ }^{7}$ In this study, the inflammatory cell infiltration in the synovium at 1 and 3 days after zymosan injection was significantly suppressed in the LOX-1 KO mice compared to that in the WT mice. No significant difference in inflammatory cell infiltration score at day 7 was found between the WT and LOX-1 KO mice. LOX-1 may be involved in the infiltration of inflammatory cells in the acute phase rather than in the chronic phase in murine ZIA model. Additionally, although the LOX-1 expression was observed in that of the WT mice, no expression of LOX-1 was observed in the synovial vascular endothelium of the LOX-1 KO mice during the entire experimental period after zymosan injection. Therefore, these results suggest that LOX-1 expression in the synovial vascular endothelium may play a role in the inflammatory cell infiltration process in ZIA model mice.

Synovial membrane cells consist of macrophage type 1 cells and fibroblast-like type 2 cells. Synovial hyperplasia, which is characteristically seen in synovitis of RA, is caused by the infiltration of type 1 cells and proliferation of a fibroblast-like cell. ${ }^{29} \mathrm{~A}$ previous report showed that LOX-1 expression was detected in activated macrophages. ${ }^{30}$ Recently, Ishikawa et al. also reported the expression of LOX-1 and the presence of ox-LDL in type 1 and type 2 cells in stratified human RA synovial membrane cells, which indicates the possibility of involvement of the LOX-1/ox-LDL system in synovial hyperplasia. ${ }^{17}$ In the current study, the inflammatory cell infiltration at 1 and 3 days and the synovial hyperplasia at 3 and 7 days after the zymosan injection in the LOX-1 KO mice was significantly less than that in the WT mice. Although LOX-1 expression in the synovial cells was observed in the WT mice, no LOX-1 expression was observed in synovial cells of the LOX-1 KO mice during the entire experimental period after zymosan injection. In the present study, we found a discrepancy between the peak times of inflammatory cell infiltration and occurrence of synovial hyperplasia as synovial hyperplasia occurs following inflammatory cell infiltration in general. This time discrepancy was reported in previous studies, ${ }^{7,18}$ as well as in this study. These findings suggest that inflammatory cell infiltration including macrophages was initially suppressed in the LOX-1 KO mice, and that synovial hyperplasia was suppressed secondarily. From these findings, we could also conclude that the LOX-1/ox-LDL system plays a role in synovial hyperplasia of ZIA model mice.

LOX-1 expression has been detected in human RA cartilage. ${ }^{10}$ Previous reports also showed the existence of ox-LDL in the synovial fluid of RA. ${ }^{31}$ Furthermore, the binding of LOX-1 to ox-LDL is thought to suppress the generation of ROS through the activation of NF- $\kappa B$ and inhibition of the PI 3 kinase/Akt pathway, which causes nonapoptotic cell death. ${ }^{12,32}$ In the current study, cartilage degeneration in the LOX-1 KO mice was significantly inhibited compared to that in the WT mice at 7 days after zymosan injection. Moreover, although the expression of LOX-1 and ox-LDL was observed in the chondrocytes of the WT mice during the entire experimental period after zymosan injection, no LOX-1 or ox- 


\section{Zymosan}

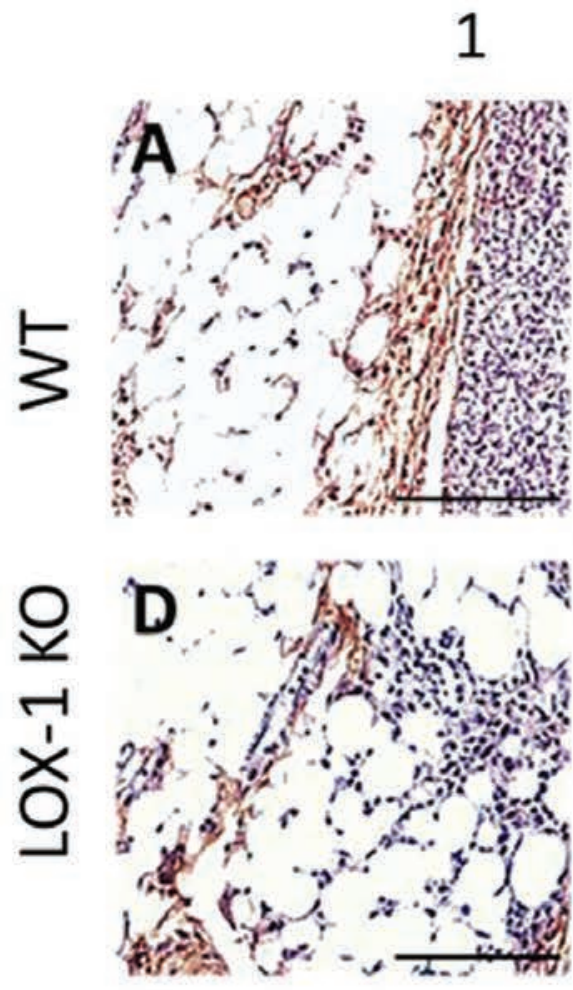

3

7 (days)
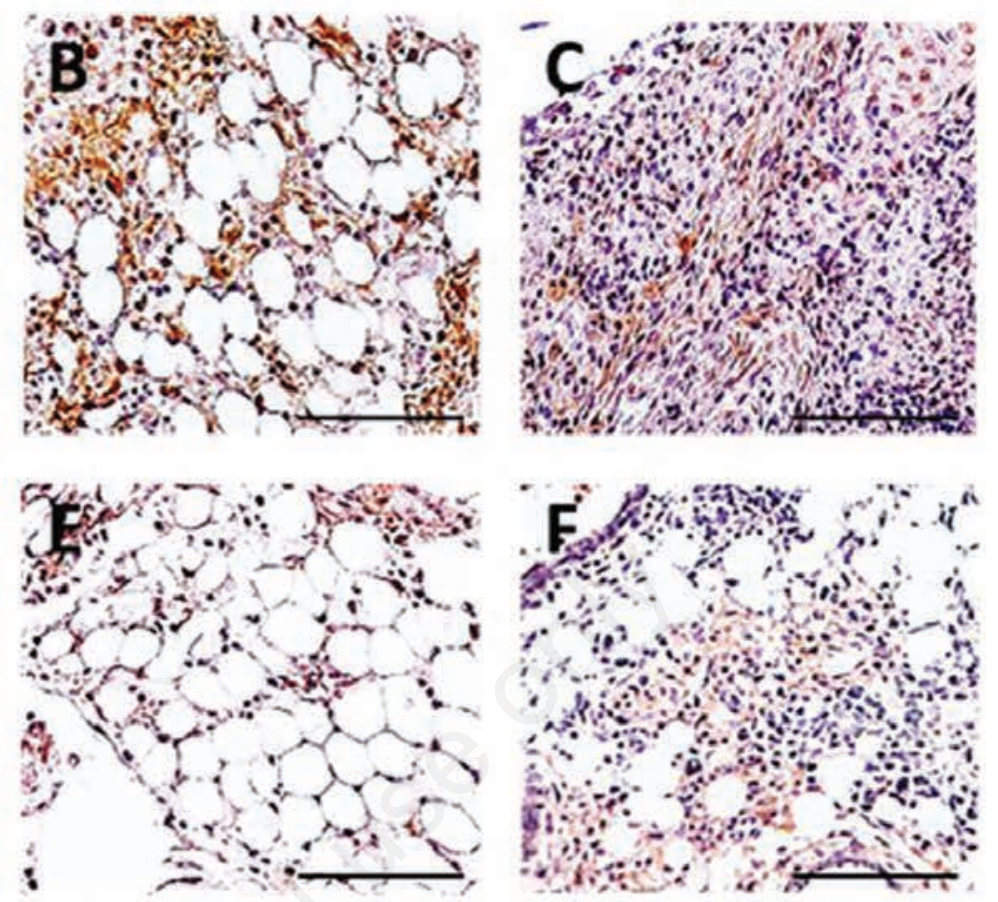

G

(Score)

\section{MMP-3 positive score}

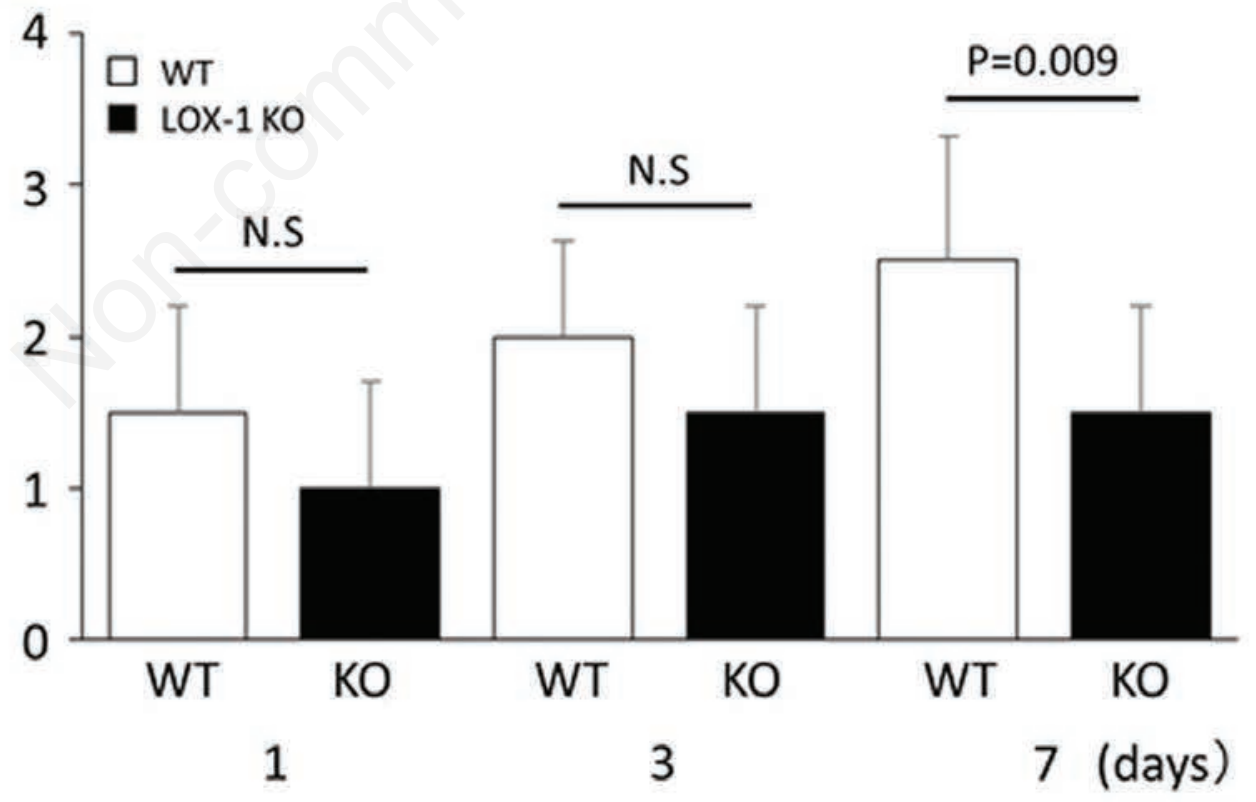

Figure 7. The panels show representative synovial tissues with immunostaining of MMP-3 (A-F). MMP-3 expression in the synovial cells of WT mice at day 1, 3 and 7 after zymosan injection (A-C) at 200x magnification. MMP-3 expression in the synovial cells of LOX-1 KO mice at day 1, 3 and 7 after zymosan injection (D-F). MMP-3 positive cells are observed both in synovial cells of WT (A-C) and LOX-1 KO mice (D-F) during all the experience time. At day 7, MMP-3 in synovium of LOX-1 KO mice (F) is stained weaker than in that of WT mice (C). The graphs show the positive cell score of MMP-3 expression in the synovium of WT and LOX-1 KO mice after zymosan injection at each experimental time $(G)$. The antibodies used were rabbit anti-mouse MMP-3 polyclonal antibody. Data are presented as mean \pm SD ( $n=10$, in each group); $\mathbf{P}<\mathbf{0 . 0 5}$ was regarded as a significant difference (Student's t-test). N.S, not significant. Scale bars: $100 \mu \mathrm{m}$. 
LDL expression was observed in the chondrocytes of LOX-1 KO mice. These findings suggest that the LOX-1/ox-LDL system plays various roles in cartilage degeneration in ZIA model mice.

The destruction of articular cartilage in arthritis is mediated mainly by a series of proteinases. Among these, the MMPs, especially MMP-1 and MMP-3, play pivotal roles in the degradation of cartilage matrices in arthritis. Previous studies reported that MMP-1 and MMP-3 expression was enhanced by the stimulation of ox-LDL in the synovial fibroblast-like cells of human RA. ${ }^{17}$ In this study, MMP-3 expression was observed in the synovial cells of the WT mice during the entire experimental period after zymosan injection. MMP-3 expression

\section{Zymosan}
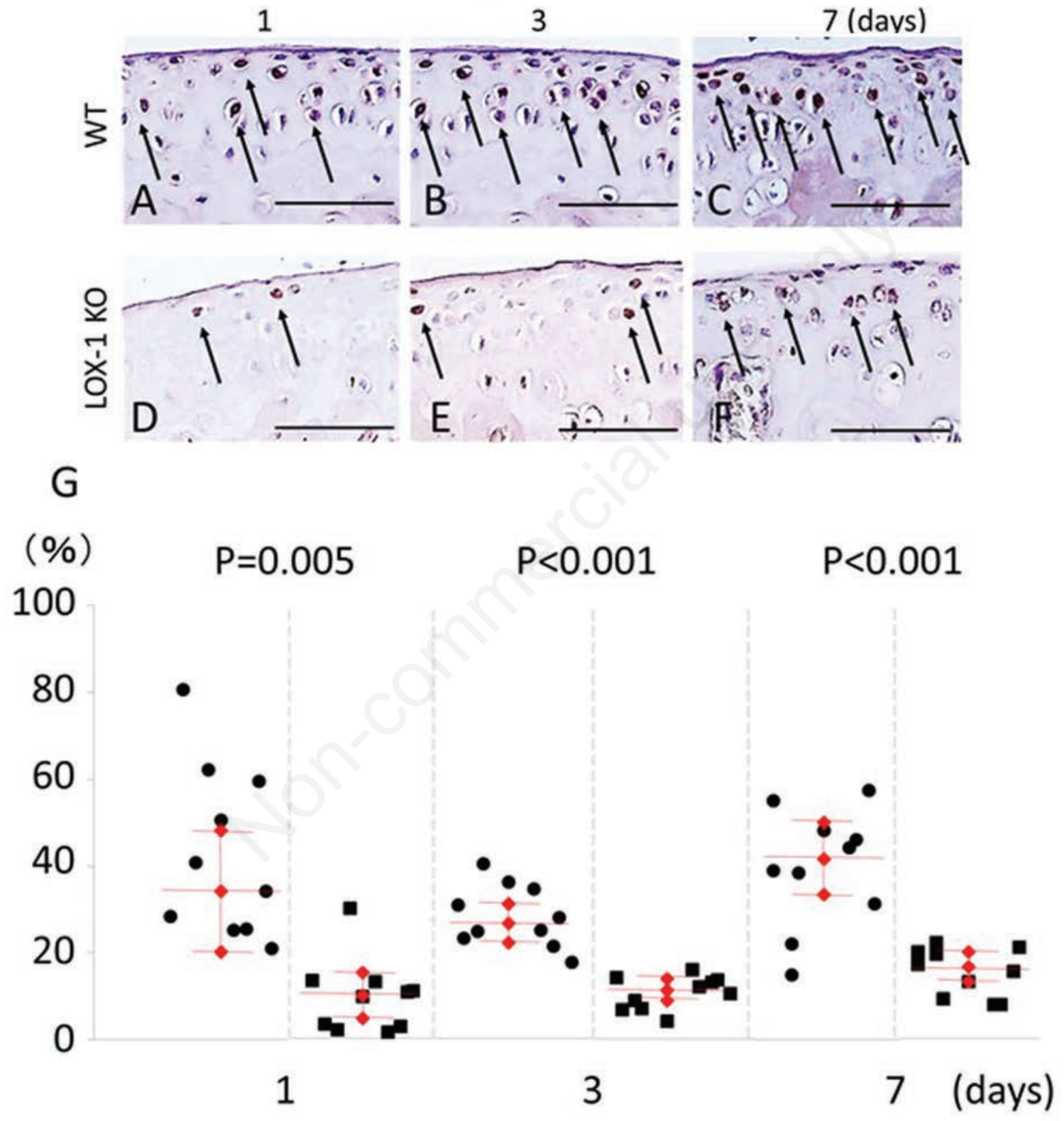

Figure 8. The panels show representative tibial chondrocyte immunostaining of MMP-3 (A-F). MMP-3 expression in the chondrocyte of WT mice at day 1,3 and 7 after zymosan injection (A-C) at $400 \times$ magnification. MMP-3 expression in the chondrocyte of LOX-1 KO mice at day 1, 3 and 7 after zymosan injection (D-F). MMP-3 positive cells are observed both in chondrocyte of WT (A-C) and LOX-1 KO mice (D-F) during all the experimental time. MMP-3 in chondrocytes of LOX-1 KO mice (D-F) is stained weaker than in that of WT mice (AC) during all the experimental time. The graphs show the positive cell score of MMP-3 expression in the chondrocytes of WT and LOX-1 KO mice after zymosan injection at each experimental time (G). Arrows show the MMP-3 positive chondrocytes. The antibodies used were rabbit anti-mouse MMP-3 polyclonal antibody. Scale bars: $100 \mu \mathrm{m}$. 
in the synovial cells of the LOX-1 KO mice was significantly less than in the synovial cells of the WT mice at 7 days after zymosan injection. These findings suggest that the LOX-1/ox-LDL system is involved in the induction of MMP-3 in the synovial cells of ZIA model mice. In the current study, there was no clear expression of MMP-1 in synovial cells and chondrocytes of the WT or the LOX-1 KO mice (data not shown), which suggests that MMP-3 may be a more significant mediator of arthritis development than MMP-1 in ZIA model mice. Further research is necessary to determine which specific MMPs are associated with cartilage degeneration in ZIA model mice.

A previous report has also shown that ox-LDL-mediated by LOX-1 enhance MMP-3 production in articular chondrocytes. ${ }^{10}$ In this study, MMP-3 expression was observed in the chondrocytes of both the WT and LOX-1 KO mice with ZIA. Additionally, the MMP-3 positive cell rate of articular chondrocytes was significantly lower in the LOX-1 KO mice than in the WT mice during the entire experimental period. These findings indicate that ox-LDL binding to LOX-1 may induce MMP-3 expression in chondrocytes, after which cartilage matrix destruction will develop in ZIA model mice. To summarize these findings, two mechanisms of cartilage degeneration in the murine ZIA model must be considered (Figure 9). In one mechanism, cartilage degeneration may be caused by the MMP-3 induced by the LOX-1/ox-LDL system in the synovial cells. In the other mechanism, the LOX-1 and ox-LDL expressed in chondrocytes may induce the MMP-3, which leads to the development of cartilage degeneration. An interesting aspect of this mechanism is that cartilage degeneration may develop via the mediation of both synovial cells and chondrocytes. Although a significant difference in inflammatory cell infiltration was found between the WT and LOX-1 KO mice at days 1,3 , and 7 , a significant difference in cartilage degeneration was observed at day 7. Three causes of this time discrepancy may be considered. First, in the inflamed synovium, proteolytic enzymes such as MMP-3 will flow out into synovial fluid, and then the cartilage matrix is destroyed. Second, in chondrocytes, proteolytic enzymes such as MMP-3 will be released after flow of ox-LDL into synovial fluid penetrates chondrocytes and binds to LOX1. Then, cartilage degeneration will occur. Third, TNF- $\alpha$ has been suggested to remain in synovial tissue such as synovial lining cells in the late phase of inflammation as previously described. ${ }^{33}$ In the present study, we found a significant difference in syn- ovial hyperplasia between the WT and LOX-1 KO mice at day 7. Proteolytic enzymes such as MMP-3 released from hyperplastic synovium might cause a significant difference in cartilage degeneration between the WT and LOX-1 KO mice. The time discrepancy described above might be involved in the fact that the significant difference in cartilage degeneration of both mice observed at day 7. The time discrepancy between inflammatory cell infiltration and cartilage destruction was also reported in a previous study, ${ }^{7}$ as well as the present study. The current study has some limitations. First, in this study, arthritis was induced in the right knees of mice via zymosan injection, and saline was injected into the left knees. Pro-inflammatory cytokines produced by zymosan injection might cause minimal changes in the left knee by systemic circulation. To eliminate the systemic effect of local zymosan injection completely, it might be necessary to divide the mice into the zymosan injection group and the control group. However, in a published study, ${ }^{7}$ where the zymosan-injected knee was compared with the contralateral saline-injected knee in the same mouse, arthritic change was observed slightly in the control knee. Since we have also found the minor arthritic changes in the control left knee of the current study, the influence of pro-inflammatory cytokines via systemic circulation might be very low. The second, the mechanism connecting arthritis development with the LOX-1/ox-LDL system was not completely clarified. Although our results suggest that arthritis may increase the function of the LOX-1/ox-LDL system, further studies are necessary to identify a direct correlation. The third limitation of the current study was the lack of insight concerning the origin of ox-LDL expression. However, we considered three possible modes of association between ox-LDL and articular chondrocytes. First, ox-LDL in the systemic circulation might enter the joint fluid through the synovial capillary. The third, LDL in the systemic circulation might enter the joint fluid and be oxidized in an inflamed joint cavity. Third, LDL penetrating the degraded cartilage matrix might become oxidized by ROS produced by articular chondrocytes. Chondrocytes produce ROS under inflammatory environment as previously described, ${ }^{34,35}$ but it was hard to determine the timing of oxidation with this study design. Since ox-LDL is a large protein of approximately $550 \mathrm{kDa},{ }^{36}$ it is unlikely that ox-LDL penetrates the extra-

\section{Zymosan to synovial fluid}

\section{Synovial macrophage activation and Leukocyte infiltration}

Pro-inflammatory cytokines

\section{LOX-1 expression in synovial vascular endothelium}

$$
\downarrow \text { ox-LDL }
$$

\section{Leukocyte rolling, adhesion and infiltration}

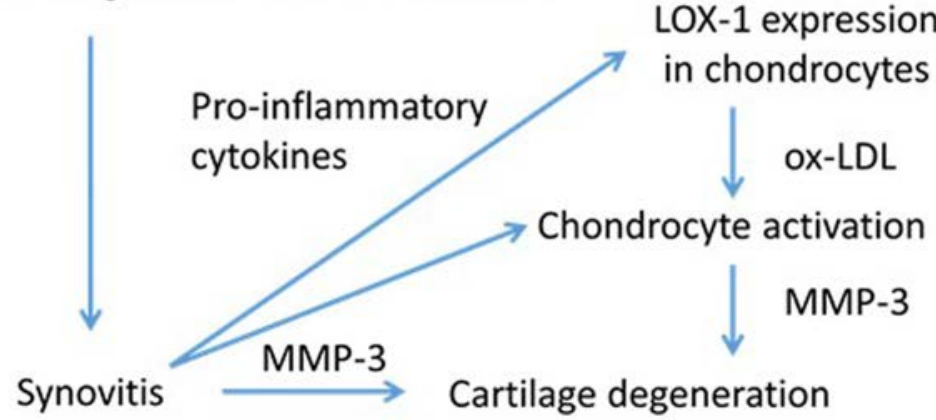

Figure 9. Flow chart summarizing the results of the present study and the assumed relevance of the oxidized low-density lipoprotein (ox-LDL)/lectin-like ox-LDL receptor 1 (LOX-1) system to the pathogenesis of ZIA. The flowchart shows the activation of synovial cells and chondrocytes by LOX-1/ox-LDL system via pro-inflammatory cytokines. 
cellular matrix of normal articular cartilage to bind to the LOX-1 expressed in chondrocytes. However, degenerated cartilage is known to release high-molecular weight molecules such as keratin sulfate, chondroitin sulfate, and hyaluronic acid from the inner layer, suggesting that ox-LDL might permeate into degenerated cartilage. In this way, it is possible that oxidation occurred because of the joint inflammation in the WT and LOX-1 KO mice. Ox-LDL expression was detected in the WT mice, but not in the LOX-1 KO mice, which is likely because the ox-LDL was not internalized through the LOX-1 receptor. Further research is necessary to determine the site of LDL oxidation and the exact mechanism by which oxLDL regulates the pathogenesis of arthritis. For example, investigation using soluble LOX-1 may be necessary. ${ }^{17}$ Moreover, the generation of additional data indicating the effects of blocking LOX-1 signal with antagonists of LOX-1, small interfering RNA, or neutralizing antibodies for arthritis induction may strengthen our theory in the current study. The third limitation of the present study is that the concentrations of pro-inflammatory cytokines such as serum TNF- $\alpha$, IL- $1 \beta$, and IL- 6 were not examined. Joint inflammation in murine ZIA has been reported to depend more on IL- $1 \beta$ than on TNF- $\alpha .^{37,38}$ IL- 6 also has been reported to be involved in the early phase of ZIA. ${ }^{39}$ As no apparent inflammatory changes were observed in the contralateral saline-injected knees in the present study, expression levels of pro-inflammatory cytokines in serum might be very low, and they were expected to be observed mainly in synovial fluid. We would like to clarify the tendency and functions of inflammatory mediators in the serum and joint fluid of ZIA mice in future studies.

In conclusion, the LOX-1-mediated proinflammatory mechanism may be involved in the pathogenesis of murine ZIA. This indicates that the treatment of abnormal lipid metabolism may contribute to the prevention and suppression of arthritis.

\section{References}

1. Zhang PY, Xu X, Li XC. Cardiovascular diseases: oxidative damage and antioxidant protection. Eur Rev Med Pharmacol Sci 2014;18:3091-6.

2. Sawamura T, Kume N, Aoyama T, Moriwaki H, Hoshikawa H, Aiba Y, et al. An endothelial receptor for oxidized low-density lipoprotein. Nature 1997;386:73-7.

3. Kume N, Murase T, Moriwaki H, Aoyama T, Sawamura T, Masaki T, et al. Inducible expression of lectin-like oxidized LDL receptor-1 in vascular endothelial cells. Circ Res 1998;83: 322-7.

4. Aoyama T, Fujiwara H, Masaki T, Sawamura $T$. Induction of lectin-like oxidized LDL receptor by oxidized LDL and lysophosphatidylcholine in cultured endothelial cells. J Mol Cell Cardiol 1999;31:2101-14.

5. Hofnagel O, Luechtenborg B, Stolle K, Lorkowski S, Eschert H, Plenz G, et al. Proinflammatory cytokines regulate LOX-1 expression in vascular smooth muscle cells. Arterioscler Thromb Vasc Biol 2004;24:1789-95.

6. Kume N, Moriwaki H, Kataoka H, Minami M, Murase T, Sawamura T, et al. Inducible expression of LOX-1, a novel receptor for oxidized LDL, in macrophages and vascular smooth muscle cells. Ann N Y Acad Sci 2000;902: 323-7.

7. Nakagawa T, Akagi M, Hoshikawa H, Chen M, Yasuda T, Mukai S, et al. Lectin-like oxidized low-density lipoprotein receptor 1 mediates leukocyte infiltration and articular cartilage destruction in rat zymosan-induced arthritis. Arthritis Rheum 2002;46: 2486-94.

8. Akagi M, Kanata S, Mori S, Itabe H, Sawamura T, Hamanishi C. Possible involvement of the oxidized low-density lipoprotein/lectin-like oxidized lowdensity lipoprotein receptor-1 system in pathogenesis and progression of human osteoarthritis. Osteoarthritis Cartilage 2007;15:281-90.

9. Nakagawa T, Yasuda T, Hoshikawa H, Shimizu M, Kakinuma T, Chen M, et al. LOX-1 expressed in cultured rat chondrocytes mediates oxidized LDLinduced cell death-possible role of dephosphorylation of Akt. Biochem Biophys Res Commun 2002;299:91-7.

10. Kakinuma T, Yasuda T, Nakagawa T, Hiramitsu T, Akiyoshi M, Akagi M, et al. Lectin-like oxidized low-density lipoprotein receptor1 mediates matrix metalloproteinase 3 synthesis enhanced by oxidized low-density lipoprotein in rheumatoid arthritis cartilage. Arthritis Rheum 2004;50:3495-503.

11. Simopoulou T, Malizos KN, Tsezou A. Lectin-like oxidized low density lipoprotein receptor 1 (LOX-1) expression in human articular chondrocytes. Clin Exp Rheumatol 2007;25:605-12.

12. Nishimura S, Akagi M, Yoshida K, Hayakawa S, Sawamura T, Munakata $\mathrm{H}$, et al. Oxidized low-density lipoprotein (ox-LDL) binding to lectin-like oxLDL receptor 1 (LOX-1) in cultured bovine articular chondrocytes increases production of intracellular reactive oxy- gen species (ROS) resulting in the activation of $\mathrm{NF}-\kappa \mathrm{B}$. Osteoarthritis Cartilage 2004;12:568-76.

13. Zushi S, Akagi M, Kishimoto $\mathrm{H}$, Teramura T, Sawamura T, Hamanishi C. Induction of bovine articular chondrocyte senescence with oxidized low-density lipoprotein through lectin-like oxidized low-density lipoprotein receptor 1. Arthritis Rheum 2009;60:3007-16.

14. Kishimoto H, Akagi M, Zushi S, Teramura T, Onodera Y, Sawamura T, et al. Induction of hypertrophic chondrocyte-like phenotypes by oxidized LDL in cultured bovine articular chondrocytes through increase in oxidative stress. Osteoarthritis Cartilage 2010;18: 1284-90.

15. Hashimoto K, Mori S, Oda Y, Nakano A, Sawamura T, Akagi M. Lectin-like oxidized low density lipoprotein receptor 1-deficient mice show resistance to instability-induced osteoarthritis. Scand J Rheumatol 2016;45:412-22.

16. Hashimoto K, Oda Y, Nakamura F, Kakinoki R, Akagi M. Lectin-like, oxidized low-density lipoprotein receptor1-deficient mice show resistance to agerelated knee osteoarthritis. Eur J Histochem 2017;61:2762.

17. Ishikawa $\mathrm{M}$, Ito $\mathrm{H}$, Akiyoshi $\mathrm{M}$, Kume $\mathrm{N}$, Yoshitomi $\mathrm{H}$, Mitsuoka $\mathrm{H}$, et al. Lectin-like oxidized low-density lipoprotein receptor 1 signal is a potent biomarker and therapeutic target for human rheumatoid arthritis. Arthritis Rheum 2012;64:1024-34.

18. Keystone EC, Schorlemmer HU, Pope C, Allison AC. Zymosan-induced arthritis: a model of chronic proliferative arthritis following activation of the alternative pathway of complement. Arthritis Rheum 1977;20:1396-401.

19. Gegout P, Gillet P, Chevrier D, Guingamp C, Terlain B, Netter P. Characterization of zymosan-induced arthritis in the rat: effects on joint inflammation and cartilage metabolism. Life Sci 1994;55:PL321-6.

20. Rosas EC, Correa LB, Pádua Tde A, Costa TE, Mazzei JL, Heringer AP, et al. Anti-inflammatory effect of Schinus terebinthifolius Raddi hydroalcoholic extract on neutrophil migration in zymosan-induced arthritis. Ethnopharmacol 2015;175:490-8.

21. Belenska-Todorova L, Gyurkovska V, Ivanovska N. How complement activation influences the development of chronic synovitis in a mouse model of rheumatoid arthritis. Scand J Rheumatol 2016;45:13-22.

22. Mehta JL, Sanada N, Hu CP, Chen J, Dandapat A, Sugawara F, et al. Deletion of $\mathrm{LOX}-1$ reduces atherogenesis in 
LDLR knockout mice fed high cholesterol diet. Circ Res 2007;100:1634-42.

23. Musumeci G, Castrogiovanni P, Mazzone V, Szychlinska MA, Castorina $\mathrm{S}$, Loreto C. Histochemistry as a unique approach for investigating normal and osteoarthritic cartilage. Eur J Histochem 2014;58:2371.

24. Krenn V, Morawietz L, Burmester GR, Kinne RW, Mueller-Ladner U, Muller B, et al. Synovitis score: discrimination between chronic low-grade and highgrade synovitis. Histopathology 2006;49:358-64.

25. Coles JM, Zhang L, Blum JJ, Warman ML, Jay GD, Guilak F, et al. Loss of cartilage structure, stiffness, and frictional properties in mice lacking PRG4. Arthritis Rheum 2010;62:1666-74.

26. de Munter W, Blom AB, Helsen MM, Walgreen B, van der Kraan PM, Joosten LA, et al. Cholesterol accumulation caused by low density lipoprotein receptor deficiency or a cholesterol-rich diet results in ectopic bone formation during experimental osteoarthritis. Arthritis Res Ther 2013;15:R178.

27. Zhang F, Wang C, Wang H, Lu M, Li Y, Feng $\mathrm{H}$, et al. Ox-LDL promotes migration and adhesion of bone marrowderived mesenchymal stem cells via regulation of MCP-1 expression. Mediators Inflamm 2013;2013:691023.

28. Fan X, Zhu JY, Sun Y, Luo L, Yan J, Yang $X$, et al. Evodiamine inhibits zymosan-induced inflammation in vitro and in vivo: Inactivation of NF- $\kappa \mathrm{B}$ by inhibiting $\mathrm{I} \kappa \mathrm{B} \alpha$ phosphorylation. Inflammation 2017;40:1012-27.

29. Fan L, Zong M, Gong R, He D, Li N, Sun LS, et al. PADI4 epigenetically suppresses p21 transcription and inhibits cell apoptosis in fibroblast-like synoviocytes from rheumatoid arthritis patients. Int J Biol Sci 2017;25;13:35866.

30. Kanaoka Y, Koga M, Sugiyama K, Ohishi K, Kataoka Y, Yamauchi A. Varenicline enhances oxidized LDL uptake by increasing expression of LOX-1 and CD36 scavenger receptors through $\alpha 7 \mathrm{nAChR}$ in macrophages. Toxicology 2017;380:62-71.

31. Dai L, Zhang Z, Winyard PG, Gaffney $\mathrm{K}$, Jones H, Blake DR, et al. A modified form of low-density lipoprotein with increased electronegative charge is present in rheumatoid arthritis synovial fluid. Free Radic Biol Med 1997;22: 705-10.

32. Nakagawa T, Yasuda T, Hoshikawa H, Shimizu M, Kakinuma T, Chen M, et al. LOX-1 expressed in cultured rat chondrocytes mediates oxidized LDLinduced cell death-possible role of dephosphorylation of Akt. Biochem Biophys Res Commun 2002;299:91-7.

33. Nagate T, Kawai J, Nakayama J. Therapeutic and preventive effects of methotrexate on zymosan-induced arthritis in SKG mice. J Vet Med Sci 2009;71:713-7.
34. Xue EX, Lin JP, Zhang Y, Sheng SR, Liu HX, Zhou YL, et al. Pterostilbene inhibits inflammation and ROS production in chondrocytes by activating Nrf2 pathway. Oncotarget 2017;8:4198842000.

35. Henrotin YE, Bruckner P, Pujol JP. The role of reactive oxygen species in homeostasis and degradation of cartilage. Osteoarthritis Cartilage 2003;11:747-55.

36. Fong LG, Parthasarathy S, Witztum JL, Steinberg D. Nonenzymatic oxidative cleavage of peptide bonds in apoprotein B-100. J Lipid Res 1987;28:1466-77.

37. Van de Loo FAJ, Joosten LAB, van Lent PLEM, Arntz OJ, van den Berg WB. Role of interleukin-1, tumor necrosis factor, and interleukin-6 in cartilage proteoglycan metabolism and destruction: effect of in situ blocking in murine antigen- and zymosaninduced arthritis. Arthritis Rheum 1995;38:164-72.

38. Pettipher ER, Salter ED. Resident joint tissues, rather than infiltrating neutrophils and monocytes, are the predominant sources of TNF-in zymosan-induced arthritis. Cytokine 1996;8:130-3.

39. Rosas EC, Correa LB, Pádua Tde A, Costa TE, Mazzei JL, Heringer AP, et al. Anti-inflammatory effect of Schinus terebinthifolius Raddi hydroalcoholic extract on neutrophil migration in zymosan-induced arthritis. J Ethnopharmacol 2015;175:490-8. 